Balbina Rajchelt*

\title{
DIE FORTSCHREITENDE MULTIKULTURALITÄT IM DEUTSCHEN SPRACHRAUM UND DER EINFLUSS VON GLOBALISIERUNG UND MIGRATION AUF DAS UMGANGSDEUTSCHE
}

Deutschland ist ohne Zweifel ein Migrationsland. Die Mittlerweile etwa 20 Millionen Einwohner mit Migrationshintergrund machen den deutschsprachigen Raum zu einem Schmelztiegel verschiedener Kulturen und Sprachen. Dies bewirkt eine rasante Entwicklung und Anpassung der deutschen Sprache auf diese neuen Bedingungen; und nirgends sind diese Veränderungen sichtbarer als in der Umgangssprache.

Der folgende Beitrag befasst sich mit der Entwicklung der deutschen Umgangssprache im Laufe der letzten Jahrzehnte, vor allem im Hinblick darauf, wie diese sich durch die stetig wachsende Zahl an Migranten aus anderen Sprachräumen verändert hat. An Beispielen verschiedener Ausdrücke und Phrasen, die sich durch den Einfluss anderer Sprachen und Kulturen im modernem Umgangsdeutsch etabliert haben, werden bestimmte Trends in der Sprachentwicklung präsentiert. Ebenso werden migrationsbedingte Veränderungen in der Grammatik des Deutschen dargestellt und begründet.

Des Weiteren wird der Einfluss der Globalisierung auf dass Umgangs- und Allgemeindeutsche anhand kurzer Beispiele vorgestellt und analysiert.

Letztlich wird anhand der vorgestellten Beispiele und Trends eine Prognose für die mögliche zukünftige Entwicklung des Umgangs- und zwangsläufig auch des Allgemeindeutschen erstellt und die Frage beantwortet, ob diese Veränderungen für die Sprache förderlich, oder eher schädlich sind und ob es lohnenswert und überhaupt möglich ist, ihnen entgegenzusteuern.

Das Konzept der Multikulturalität wird heutzutage vorwiegend im sozialen Kontext, als Koexistenz mehrerer Kulturgruppen Beschrieben. Dem Duden nach handelt es sich hierbei um „Das Vorhandensein von Einflüssen mehrerer

Mgr Balbina Rajchelt, Uniwersytet Łódzki. 
Kulturen, kulturelle Vielfalt“ (Duden). Dieses Phänomen betrifft jedoch die linguistische Ebene ebenso, wie die ökonomisch-soziale. Die Multikulturalität der deutschen Sprache, die mit der fortschreitenden Multikulturalisierung Deutschlands einhergeht, ist kein neuer Prozess, sondern eine weitere, rasant beschleunigte Etappe eines Prozesses, der schon seit Jahrhunderten andauert. Völkerwanderungen und Migrationen waren schon immer ein Teil der gesellschaftlichen Entwicklung Europas und die damit verbundenen Sprachentwicklungen und Sprachveränderungen üben damals wie heute einen starken Einfluss auf unsere Kommunikation aus. Ein gutes Beispiel hierfür bildet das Englische, welches sich als Mischung germanischer, keltischer, französischer und nordischer Einflüsse $\mathrm{zu}$ einer einzigartigen, international-geprägten Sprache entwickelt hat. Auch das moderne Deutsch entwickelte sich als Mischung intralingualer Einflüsse (Dialekte, Mundarten) und außersprachlicher Einflüsse. Dennoch muss betont werden, das die deutsche Sprache, seit den 60-er Jahren enormen Veränderungen unterliegt. Diese Veränderungen sind zu einem großen Teil dem enormen Zufluss an Migranten in Deutschland zuzuschreiben. Mittlerweile leben in Deutschland fast 20 Mio. Menschen mit Migrationshintergrund (fast ein Viertel der Gesamtbevölkerung). Diese Millionen Ausländer brachten ihren eigenen Sprach- und Wortschatz mit sich, der nun einen entscheidenden Einfluss auf die weitere Entwicklung der deutschen Sprache nimmt.

Das tägliche Zusammenleben von Deutschen und Ausländern und die Wirkung mehrerer Sprachen auf die Kommunikation im schulischen, beruflichen und privaten Alltag führte im Laufe der Zeit, insbesondere seit den 90-er Jahren, zur Entwicklung verschiedener vorwiegend umgangssprachlicher Varietäten, wie z.B. Kiezdeutsch, Türkisch-Deutsch, oder Russisch-Deutsch, die vor allem in Großstätten zu hören sind und von Sprach-Puristen oftmals als Verunglimpfung der deutschen Sprache, Sprachverfall, oder gar Vaterlandsverrat gesehen werden. ${ }^{1}$ Andere sind der Ansicht, es handele sich hierbei lediglich um eine ganz gewöhnliche Etappe der sprachlichen Entwicklung, Migrationssprachen hätten keinen Einfluss auf das Standarddeutsche, Deutschland sei schon immer ein multikulturelles Land gewesen, es gäbe da also gar nichts neues (Hinrichs 2013: 70). Der Leipziger Sprachwissenschaftler Uwe Hinrichs (2013: 77) beteuert, dies sei lediglich die zukünftige sprachliche Realität: „Noch nie habe es in der Weltgeschichte eine Sprache gegeben, in deren Umkreis viele andere Sprachen dazugekommen sind und sich keine massiven sprachlichen Veränderungen ergeben haben."

Vor allem die Großstadtregionen werden so zu einem Schmelztiegel des linguistischen Austausches. Da nun knapp ein Viertel der Bevölkerung Deutschlands einen Migrationshintergrund hat, sind oft zweisprachige Sätze oder

1 s. a. Arnold, Ronny (2012), Wie Migration die deutsche Sprache verändert, unter: https://p.dw.com/p/15exb [Zugriff am 12.12.2019]. 
Ausdrücke zu hören, in denen der Sprecher innerhalb eines Satzes vom Deutschen in eine andere Sprache hinüberwechselt. Dies gilt vor allem für die am häufigsten in Deutschland vertretenen Migrationssprachen:

Russisch:

- „Vchera v Berline proshel evangelischer Kirchentag“ („Gestern fand in Berlin der evangelische Kirchentag statt") (Hinrichs 2013: 88)

- „Mne nuzhna yeshio medizinische Begründung“ („Ich brauche noch eine medizinische Begründung“) (Hinrichs 2013: 89)

- „Dass müsst ihr regeln mit Hausmeisterom“ („das müsst ihr mit dem Hausmeister regeln“) (Hinrichs 2013: 89)

und Türkisch:

- „Plötzlich haben wir alle sağa baktık.“ („Plötzlich haben wir alle nach rechts gesehen") (Hinrichs 2013: 112)

- „Yalla, bevor der Bademeister Stress yapmadan önce.“ („Los geht's, bevor der Bademeister wieder Stress macht.") (Hinrichs 2013: 114)

- „Wir sehen uns Urlaubden sonra“ („Wir sehen uns nach dem Urlaub“). (Hinrichs 2013: 110)

Anhand dieser Sätze sind einige Trends in der Entwicklung der Migrationsdialekte in Deutschland zu erkennen. Wenn Deutsch als Zweitsprache auftritt, werden in Sätzen grammatische und lexikalische Mischformen verwendet, bei denen der Sprecher bestimmte deutsche Begriffe durch Begriffe aus seiner Muttersprache ersetzt, da er die deutschen Äquivalente nicht kennt oder sie nicht grammatisch korrekt einzusetzen weis. Viele Sprachwissenschaftler betrachten solche Strukturen einfach als Fehler, die von unzureichenden Sprachkenntnissen zeugen, selbst jedoch keinen wahrnehmbaren Einfluss auf das Standarddeutsche ausüben. Engere Integration mit dem deutschen Sprach- und Kulturraum würde $\mathrm{zu}$ einem besseren Zweitsprachenerwerb führen und so solche Mischformen abschaffen.

Der Kölner Sprachwissenschaftler Hartmut Esser (2006: 19) meint, kompetente Bilingualität bleibt die Ausnahme:

(...) International überwiegt über die Einwanderergenerationen hinweg eine deutliche Tendenz zur monolingualen Assimilation. Eine kompetente Zweisprachigkeit, also die Beherrschung von sowohl Herkunfts- als auch Landessprache auf hohem (mündlichem und schriftlichem) Niveau, wird der Ausnahmefall bleiben. Denn Bedingungen, die den Zweitspracherwerb fördern, wirken zumeist einer Beibehaltung und kompetenten Beherrschung der Muttersprache entgegen - und umgekehrt: Je höher etwa das Einreisealter und je stärker die Einbindung in den ethnischen Kontext, umso eher wird die Muttersprache beibehalten, aber umso weniger kommt es zum Zweitspracherwerb. (...)

Im Migrantendeutsch sind solche sprachlichen Mischformen jedoch bereits zum Standard geworden, da sie zweisprachigen Sprechern eine bequeme Lösung 
für das Problem von Unverständlichkeiten in der deutschen Sprache und Grammatik bietet: Code-Switching, den Wechsel zwischen zwei sprachlichen Codes, sodass der Sprecher die für ihn jeweils bequemere Version eines Ausdrucks aus einer der beiden Sprachen wählen kann. (Göttert 2013: 29)

Hinrichs (2013: 114) sieht dieses Code-Switching als ein Werkzeug zur Sprachoptimierung und als Ursache für bestimmte Veränderungen im modernen Umgangsdeutsch. Diese Veränderungen betreffen sowohl die lexikalische, wie auch die grammatikalische Sprachebene. Einerseits kommen Begriffe wie „Yalla!“ (Türkisch für „Los!“) oder „Lan“ (Türkisch für „Typ, Kumpel“) vor allem unter Jugendlichen immer häufiger auch außerhalb der Migrantengruppen vor, andererseits weisen Deutschsprecher mit Migrationshintergrund oftmals die Tendenz dazu auf, bestimmte deutsche grammatische Strukturen durch solche aus ihrer Muttersprache zu ersetzen. Diese Sprachpraktik führt zu einer steigenden Tendenz zur Vereinfachung der gebrauchten Sprache und zum Weglassen, oder Ersetzen komplizierterer Strukturen durch einfachere, ohne große Rücksicht auf grammatische Korrektheit. Hinrichs (2013: 129) meint, dieses Phänomen betrifft das Deutsche als Zweitsprache ebenso stark wie die Muttersprache des Sprechers:

Überall, wo mindestens zwei Sprachgruppen aufeinander treffen, ist der Trend zur Vereinfachung zu beobachten. Alles, was die Sprache unnötig kompliziert macht, wird weggelassen. Wenn Mehrsprachigkeit dominiert, wird alles beseitigt, was man für die Verständigung nicht braucht. Komplizierte Grammatik wird daher abgebaut und die Strukturen vereinfachen sich - übrigens nicht nur im Deutschen, sondern auch in der Muttersprache der Migranten. ${ }^{2}$

Diese Vereinfachungen betreffen in erster Linie die Fälle der deutschen Sprache, also die Kasus. Da oft auch außerhalb der Migrationsgruppen die Kasus im Umgangsdeutschen oftmals inkorrekt gebraucht werden, oder wie der Genitiv, fast gänzlich verschwinden, ist es nicht verwunderlich, dass Migranten, angesichts dieser Unsicherheit auf die jeweils passenden Kasusstrukturen aus ihren jeweiligen Muttersprachen zurückgreifen (Bellos 2013: 63).

Einige Beispiele für solche veränderten Kasusstrukturen wären, nach Hinrichs (2013: 140):

Genitiv:

- „Das Auto von meinem Vater“/“meinem Vater sein Auto“ (statt „Das Auto meines Vaters")

- „Ich habe es ihn versprochen“ (statt „ihm“)

- „Ich sprach mit den Nachbar“ (statt „dem“)

- „Hier werden Sie geholfen“ (statt „Hier wird Ihnen...“)

2 s. a. Panthöfer, Sonja (2014), Einfluss von Migrantensprachen - Multi-Kulti-Deutsch, https://www.goethe.de/de/spr/mag/20363974.html [Zugriff am 12.12.2019]. 
Dativ:

- „wir haben es den Wähler versprochen“ (statt „den Wählern“)

- „wir hörten es von den deutschen Schwimmer" (statt „Schwimmern")

- „Der Strom fließt direkt zu den Verbraucher“ (statt „Verbrauchern“)

Akkusativ:

- „Er investiert in neuen Plänen“ (statt „in neue Pläne“)

- „Sie hat darauf ein Anspruch“ (statt „einen Anspruch“)

- „Es gibt ein begabter Regisseur" (statt „einen begabten Regisseur“)

Sichtbar ist, dass im Zweifelsfall bei den Kasusformen oft auf den Akkusativ und den Nominativ (je nachdem, ob die jeweilige Muttersprache Kasus hat, oder nicht) zurückgegriffen, während die Dativ- und vor allem die Genitivformen immer weiter aus dem Usus gedrängt werden.

Auch Wortendungen und Artikel werden im Migranten- und Umgangsdeutsch oftmals verwechselt oder weggelassen, sodass Strukturen entstehen wie:

- „Ich kaufe Auto“ (statt „Ich kaufe ein Auto“)

- „Er lebt auf dem großen Fuß.“ (statt „Er lebt auf großem Fuß.“)

- „Die Bedeutung Deutschland in der Welt“ (statt „Deutschlands“)

- „Die Höhe des Betreuungsgeld“ (statt „Betreuungsgeldes“)

- „Die Breite des Spektrum“ (statt „Spektrums“)

Die Veränderungen im Gebrauch von Artikeln sind hier öfters dadurch zu erklären, dass die meisten Migrantensprachen keine Artikel Besitzen, wie etwa das Russische oder das Kroatische (Hinrichs 2013: 160).

Auch der Gebrauch von Präpositionen, die in verschiedenen Sprachen anders eingesetzt werden, gerät im Umgangsdeutschen immer mehr ins Wanken:

- „Die Zukunft für Deutschlands Banken (statt „Die Zukunft der Banken Deutschlands")

- „Wir haben eine Tendenz zur Spaltung“ (statt „Wir haben eine Tendenz der Spaltung")

- „Das Buch von meinem Vater“ (statt „Das Buch meines Vaters“)

- „Ich entsinne mich an die Rede“ (statt „Ich entsinne mich der Rede“)

- „Ich schäme mich für den Ausdruck“" (statt „Ich schäme mich des Ausdrucks“

- „Ich bin begeistert über den Film“ (statt „...von dem Film“)

- „Ersetzen Sie das Dokument mit dem Neuen.“ (statt „...durch das Neue.“)

- „Die Regierung drängt zu neuen Sanktionen“ (statt ,auf neue Sanktionen“)

Neben den bereits genannten Migrationssprachen übt selbstverständlich auch das Englische, die internationale Lingua Franca unserer modernen, globalisierten Gesellschaft, einen starken Einfluss auf die deutsche Sprache aus. Die fortwährende Globalisierung unserer Gesellschaft sorgt zusammen mit dem technologischen Fortschritt dafür, dass bestimmte Begriffe, wie „Computer“, „Layout“ oder „Smartphone“ zu einem integralen Teil der modernen deutschen Sprache geworden sind, oftmals ohne dass sich äquivalente deutsche Begriffe überhaupt entwickeln konnten. 
Hinzu kommt die stetig wachsende Beliebtheit von Social-Media-Plattformen, die eine direkte, oft informelle Kommunikation unter Nutzern begünstigen, die vor allem auf kurzen, effektiven und nicht zwingend grammatisch korrekten Texten beruht. Da das mündliche Deutsch zusätzlich durch Streams, Telefonund Onlinekonferenzgespräche begünstigt wird, prophezeien Sprachwissenschaftler in der Zukunft ein immer stärkeres auseinanderleben von Hoch- und Umgangssprache im Deutschen (Hinrichs 2013: 184).

Die komplexe deutsche Grammatik wird immer mehr vereinfacht, auch wenn dies auf Kosten der sprachlichen Eindeutigkeit geschieht, was die Sprache im Nachhinein stärker vom Kontext des Satzes abhängig macht. Die Syntaktische Entwicklung des Deutschen zeigt wiederum eine klare Tendenz zu einfacheren, optimaleren Satzstrukturen und kurzen Textformen auf. Es wird also in Zukunft immer mehr darauf geachtet was gesagt wird und weniger darauf wie es gesagt wird. (Bellos 2013: 45).

Viele Skeptiker beklagen diese Entwicklung als eine Rückbildung, oder Degeneration der deutschen Sprache, die den ursprünglichen Charakter der Sprache zerstört und nur ein vereinheitlichtes, globalisiertes Pidgin-Deutsch übrig lässt. Andere sehen es als einen natürlichen Prozess der Sprachentwicklung, einen weiteren evolutionären Schritt der deutschen Sprache im europäischen und globalen sprachlich-kulturellen Kontext. Wie dem auch sei, dieser rasante Sprachwandel, dem die deutsche seit der Mitte des 20. Jahrhunderts unterliegt dauert immer noch an, wird immer schneller und kann (und sollte) nicht mehr aufgehalten oder rückgängig gemacht werden.

Die alte Sprachsituation aus den 50ern mit ihrer Einsprachigkeit und nationalen Orientierung ist heutzutage, in unserer modernen europäischen Multi-Kulti-Gesellschaft nicht nur nicht mehr möglich, sondern nicht mehr lebensfähig. Die Sprache ist und bleibt ein Werkzeug der Kommunikation und muss sich der modernen, internationalen Gesellschaft des 21. Jahrhunderts anpassen.

\section{Literaturverzeichnis}

Bellos, David (2013), Was macht der Fisch in meinem Ohr? Sprache, Übersetzen und die Bedeutung von allem. Aus dem Englischen von Silvia Morawetz. Köln.

Esser, Hartmut (2006), Migration, Sprache und Integration. In: Esser, Hartmut (Hrsg.), Arbeitsstelle Interkulturelle Konflikte und gesellschaftliche Integration. „Forschungsbilanz 4“. Berlin, s. 14-78.

Göttert, Karl-Heinz (2013), Abschied von Mutter Sprache. Deutsch in Zeiten der Globalisierung. Frankfurt am Main.

Hinrichs, Uwe (2013), Multi Kulti Deutsch. Wie Migration die deutsche Sprache verändert. München. 


\title{
Internetquellen
}

Arnold, Ronny (2012), Wie Migration die deutsche Sprache verändert, unter: https://p.dw .com/p/15exb [Zugriff am 12.12.2019].

„Multikulturalität“ auf Duden online. URL: https://www.duden.de/rechtschreibung/ Multikulturalitaet [Zugriff am 12.12.2019].

Panthöfer, Sonja (2014), Einfluss von Migrantensprachen - Multi-Kulti-Deutsch, https:// www.goethe.de/de/spr/mag/20363974.html [Zugriff am 12.12.2019].

\section{Balbina Rajchelt \\ The advancing multiculturalism in the German language area and the influence of globalization and migration on the common or colloquial German}

\begin{abstract}
Germany is undoubtedly a country heavily influenced by migration. The now approximately 20 million inhabitants with a migration background make the German language area a melting pot of different cultures and languages. This leads to a rapid development and adaptation of the German language to these new conditions; and nowhere are these changes more visible than in the common language used in everyday situations.

The article deals with the development of colloquial German language over the past few decades, especially in regard to how it has changed due to the steadily growing number of migrants from other language areas. Certain trends in linguistic development are presented using examples of various expressions and phrases that have established themselves in modern German through the influence of other languages and cultures. Migration-related changes in the German grammar are also presented and explained.

Furthermore, the influence of globalization on the common and colloquial German language is presented and analysed using short examples.

Ultimately, based on the examples and trends presented, a forecast for the possible future development of the colloquial and inevitably also of the general German language is created and the question is answered whether these changes are beneficial to its linguistic development or rather harmful and whether it is worthwhile and even possible to try to counteract them.
\end{abstract}

Keywords: Multiculturalism, linguistics, colloquial German, globalization.

Schlüsselwörter: Multikulturalität, Linguistik, Umgangsdeutsch, Globalisierung. 